\title{
Redirection of Lamb Waves for Structural Health Monitoring
}

\author{
W. H. Ong and W. K. Chiu \\ Department of Mechanical and Aerospace Engineering, Monash University, Clayton, VIC 3800, Australia \\ Correspondence should be addressed to W. H. Ong, wern.ong@monash.edu
}

Received 31 August 2011; Accepted 7 October 2011

Academic Editor: Tao Li

Copyright ( 12012 W. H. Ong and W. K. Chiu. This is an open access article distributed under the Creative Commons Attribution License, which permits unrestricted use, distribution, and reproduction in any medium, provided the original work is properly cited.

Currently, structures are designed without structural health monitoring (SHM) in mind. It is proposed that SHM should be addressed at the design stage of new structures. This paper explores the benefit which can be gained from such considerations. The scope encompasses Lamb-wave-based SHM and a given fatigue critical location (FCL). Optimization is performed using specialised ray tracing. A case study is carried out using a specimen that simulates a hard-to-inspect region in a fuel vent hole in wings structures of aircraft. This work will report on the potential use of the focussing of stress wave to improve detectability of defect in this hard-to-inspect location. Following optimization, results are produced numerically and experimentally. The results revealed sensitivity to damage is nearly doubled while minimum detectable damage size is significantly decreased. As a result, this study brings together an assortment of specialised tools to form a workflow ready for implementation.

\section{Introduction}

Propagating Lamb-wave-based structural health monitoring methodologies have been widely reported to be convenient and efficient in detecting fatigue cracks in metallic structures $[1,2]$ and disbonds and delaminations in composite structures [3]. The propagating characteristics of Lamb waves in metals [4] and multilayered materials [5] are well documented. An attractive feature of this stress-wave-based method of structural health monitoring is that it can be used in "hard-to-reach" areas because the actuators and the sensors required can be bonded onto the structure. This is commonly known as "insitu structural health monitoring" (ISHM). The investigators of this project are amongst the many others who have contributed to the development of the Lamb-wave-based method of ISHM.

Many authors have published on optimizing and improving probability of detection (POD) and damage sensitivity. However, the works published have been focused on topics such as sensor placement, sensor selection, frequency selection, data capture, and data analysis. Such topics indicate that the structure itself is not included as a part of the optimization. SHM is not a part of the design process, rather an afterthought. A successful SHM friendly structure can be designed with less tolerance and therefore less material and weight. Consequently, the weight gained from implementing SHM systems can be offset.

A variety of tools acquired over former years have been applied to this problem. Ray tracing specialised in propagating Lamb waves has been used to redirect waves. Laser vibrometry and FEA are used to acquire displacement fields caused by Lamb waves. Damage quantification techniques described in (1) by Ong and Chiu [6] have been used to evaluate the efficacy of the structural alterations:

$$
\operatorname{Scatter}(x, y)=\frac{\sum_{t=0}^{n_{t}}\left|u_{k}(x, y, t)-u_{0}(x, y, t)\right|}{n_{t}}
$$

where $n_{t}$ is the number of time steps used, $u_{k}$ is the displacement field of the damaged specimens and $u_{0}$ is the displacement field of the flawless specimen.

A structure representing an aircraft wing spar with flange and riser has been chosen to demonstrate the benefits of wave redirection. The specimen is shown in Figure 1. Often aircraft utilize wet wings loaded with fuel; this will require a fuel vent hole to be drilled into the spar creating a stress concentration leading to fatigue cracks. Additionally, sensors cannot be placed on the riser due to the presence of fuel. 

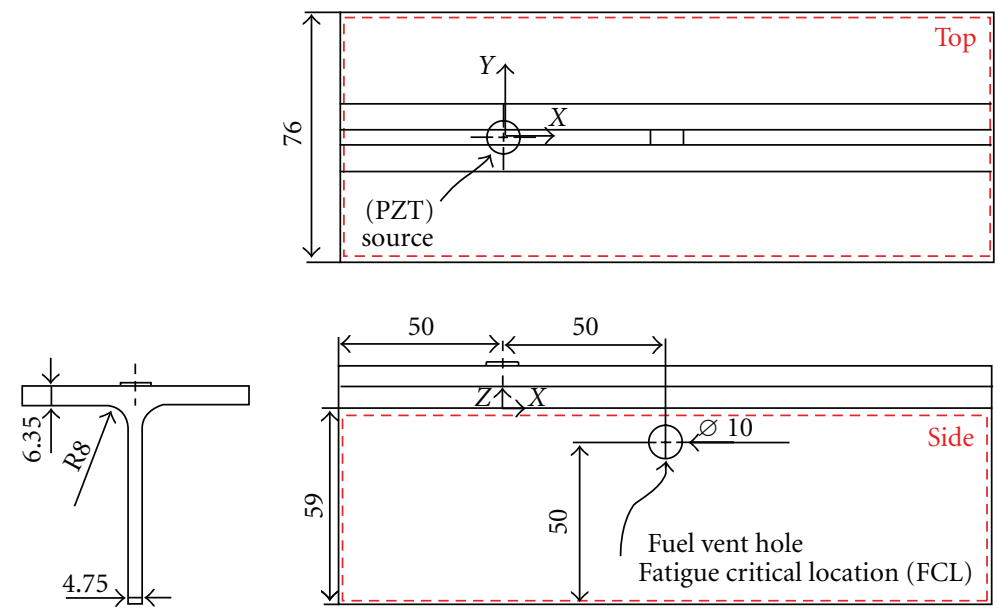

FIGURE 1: Original specimen configuration.

The condition conducive to crack growth will be a tension loading in the marked $x$ direction. This loading leads to cracks propagating perpendicular to the direction of load. Therefore, an upward (towards flange) crack and downward crack form on the fuel vent hole. The upward crack can be detected through multiple means including bulk wave and Lamb wave ultrasonics since the location has line of sight with the external facing flange. However, the line of sight with the downward crack is blocked by the fuel vent hole making the area hard to inspect. Hence, this specimen has been chosen to demonstrate wave redirection as another approach to improvement of damage detection.

Three studies performed sequentially will be presented here. The first step will be modelling the specimen with a ray tracing tool. This tool has the ability to solve the propagation of Lamb waves and present the wavefront in a clear and precise style allowing the user to determine exactly where the waves are going. Due to the simplicity of the method and equations involved, the simulations are solved in seconds allowing instant feedback and facilitation of iterative design of wave guides. The seconds step will be to test the design in finite element analysis (FEA) in order to assess the efficacy of the modifications made and confirm the predicted wave propagation in ray tracing. The final step is experimental testing of the design which provides confirmation that all the modelling performed is correct and the concept is proven and solid.

\section{Modification for Wave Redirection}

The original specimen is an aluminium $\mathrm{T}$ beam consisting of a $4.75 \mathrm{~mm}$ thick web (labelled "side") and $6.35 \mathrm{~mm}$ flange (labelled "top") as shown in Figure 1. The fuel vent hole is located on the side $50 \mathrm{~mm}$ above the bottom of the web. The focus of this work will be to detect damage in the FCL under the fuel vent hole. This $10 \mathrm{~mm}$ diameter hole is typical in aircraft structures and gives rise to stress concentrations. Lamb wave generation is handled by a piezoelectric transducer (PZT) placed on the top surface.

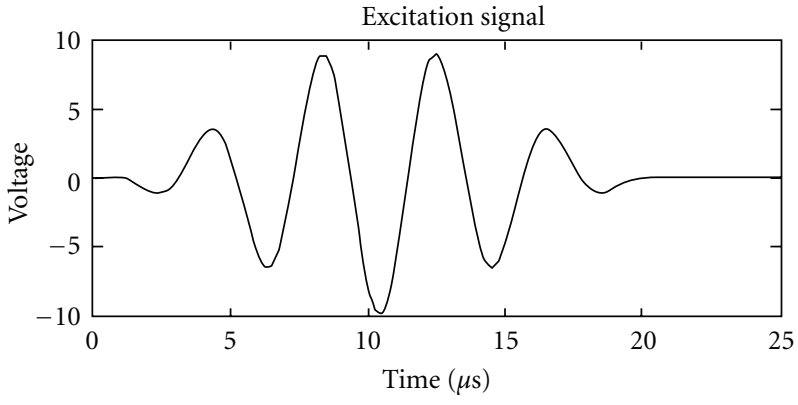

Figure 2: Hanning windowed 5-cycle sine wave excitation signal. Centre frequency set at $240 \mathrm{kHz}$.

Placement of PZTs is constrained to the top surface as the side surface will be immersed in fuel. Therefore, it will be preferable to enhance to the sensitivity to damage when the receiver is placed along the top surface. The chosen excitation signal is a $240 \mathrm{kHz} 5$-cycle Hanning windowed sine wave as shown by Figure 2. The centre frequency was chosen due resonances in the actuation system which improve the signal to noise ratio.

The modifications done to optimise SHM detectability focuses on the redirection of Lamb waves towards the fatigue critical location (FCL). In this case, the FCL is the hole drilled into the $\mathrm{T}$ beam for fuel flow in an aircraft. A circular reflector has been cut into the free edge of the $\mathrm{T}$ beam to direct the Lamb waves emitted from the PZT to the FCL. The shape was arrived at through application of ray tracing which allowed for the trial and error of many positions, sizes, and shapes. The process is described in greater detail in the ray tracing section.

An obvious problem arising from Figure 3 is the new stress concentration created by the removal of material to form the Lamb wave focusing reflector. However, the study presented here is a demonstration of concept and is not a suggestion of drastic design change. Instead, it 


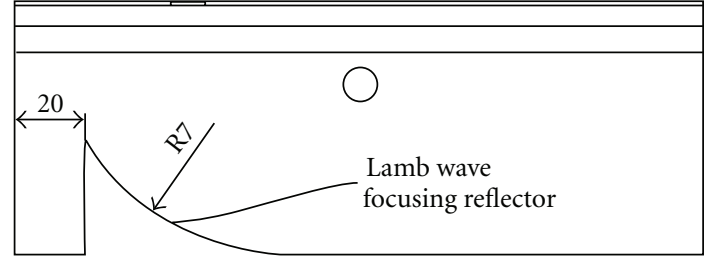

Figure 3: Modified specimen optimised for damage detection at FCL.

is recommended that existing cutouts and geometry be optimized for SHM rather removing more material.

Detection has been attempted on a $5 \mathrm{~mm}$ downward cut placed at the hole. The damage detection technique is based on the windowed scatter/difference calculations performed in a previous paper [6]. This case study demonstrates the need to have an array of specially developed tools available to optimise a structure. The scattered field calculation allows for detection of changes in the structure (such as damage) and the corresponding location for optimal detection. Ray tracing is used for fast and concise optimization of Lamb wave direction, while FEA can validate these optimizations without fabrication of a specimen.

\section{Ray Trace Study}

3.1. Method. A simple ray trace program was written in MATLAB to model Lamb wave propagation. Details and verification of the modelling technique have been published by Ong and Chiu [7]. A key difference between ray tracer used for light rays and the one presented here is the time stepping algorithm used. Typically, in the field of modelling light, the rays are solved until a set number of bounces are reached or the rays exit the work area. This results in a fast solution which solves the completed path of a ray without consideration to the transit of the rays. In the case of Lamb wave propagation in solids, the Lamb waves will never exit the workspace. Consequently, the rays will always be in transit as they are bounded within the solid. Additionally, the evolution and propagation of the rays are important; consequently, the temporal dimension has importance. As a result, the ray tracing program steps through time the same way a transient FEA simulation is done. Group velocities determine how fast the rays travel with respect to time while changes in phase velocities determine the bending of rays (refraction) at thickness change interfaces. This is calculated using Snell's law shown in (2). Reflection is assumed to be governed by (3):

$$
\frac{c_{1}}{c_{2}}=\frac{\sin \left(\theta_{1}\right)}{\sin \left(\theta_{2}\right)},
$$

where $c$ and $\theta$ theta are the phase velocity and direction of propagation, respectively. While subscripts 1 and 2 represent the entry and exit wave.

$$
\theta_{i}=\theta_{r}
$$

The side surface was chosen for ray trace modelling as this is the side which hosts the reflector and the FCL. 18 rays



Figure 4: Ray trace overlayed on displacement field captured by laser vibrometer. In addition to ray lines, 180 points were emitted to form a visualization of the wavefront.

were emitted downward from the PZT location where it was assumed Lamb waves would propagate in a radial direction despite having to go through the top surface. Effects from the $6.35 \mathrm{~mm}$ thick flange have been assumed negligible. The validity of this assumption is demonstrated by the agreement between the experimental wavefront and ray trace predicted wavefront, as shown in Figure 4. Constant duration time steps were set at 0.2 us providing a sample rate of $5 \mathrm{MHz}$, which will be identical to FEA performed later.

3.2. Results. As described earlier, the approach taken to enhancing detection of damage is to redirect Lamb wave energy. A reflector placed on the bottom edge is used for this task. The specimen was modelled in the ray trace software which gave instant feedback about the propagation of Lamb waves. This feedback was used in deciding the size and location of the reflector.

Figure 5 shows 3 models which have been solved by the ray trace software. In Figure 5(a), the original specimen has been included for comparison, showing how the waves propagate omnidirectionally. By observation, there is no direct line of sight between the FCL located at the bottom of the fuel vent hole and the PZT source. This means all the wave energy arriving at the FCL is done so through reflection (attenuated by distance and radial spread).

In Figure 5(b), a circular-shaped reflector was added to the ray trace model in the form of a boundary representing a cutout to be performed in the physical specimen. The size (radius) and location of the reflector were manipulated until the waves were focused at the desired location (FCL). Distance travelled by the reflected waves is not significantly altered; therefore, attenuation from distance is roughly the same. However, the radial spread of wave energy is converted to a gathering of energy at the focal point of the reflector. This energy will result in more reliable and sensitive detection of damage in the FCL.

Figure 5(c) shows the "FCL focus offset" model which has been included to demonstrate how the reflector parameters were arrived at. The design process was iterative through trial and error enabled by the high-speed solutions provided by ray tracing. The model which has its reflector offset $10 \mathrm{~mm}$ to the right shows the final step in iterative design before reaching optimal configuration. Sensitivity to geometry when trying to redirect energy is also revealed. 


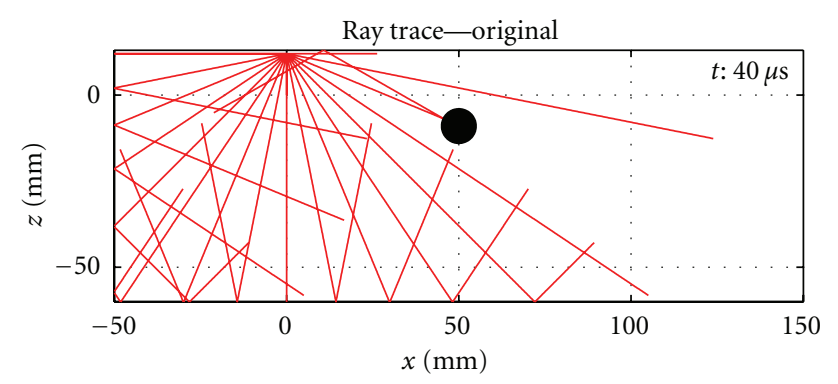

(a)

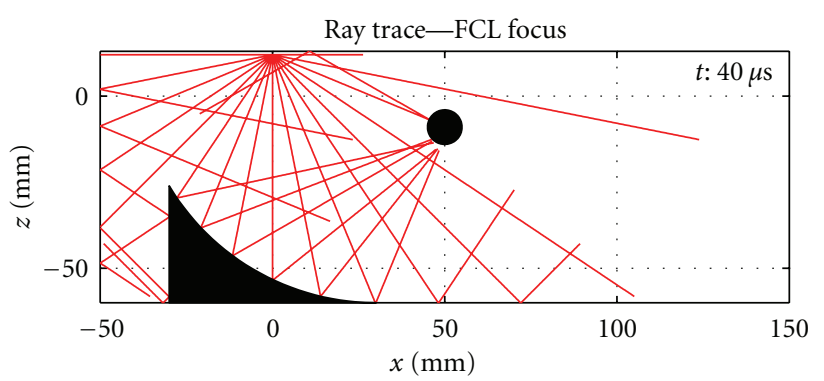

(b)

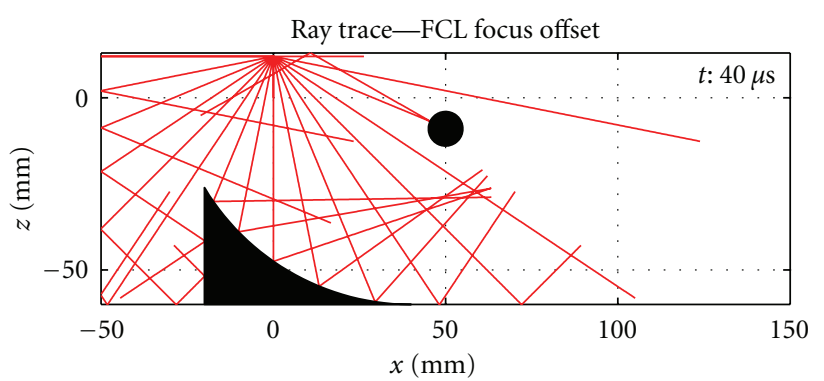

(c)

Figure 5: Ray trace snapshots.

When a reasonable focus has been achieved the dimensions are saved for use in experimental and FEA studies. This case is shown in Figure 5(b).

\section{Numerical Study}

4.1. Finite Element Setup. The specimen is modelled in 3D using tetrahedral elements. Commercially available Femap pre-/postprocessor is used in modeling, while solving is done in NEiNastran. Only out-of-plane displacements are saved to remain consistent with laser vibrometry. Further postprocessing is carried out in MATLAB to convert and analyse the displacement fields from the model. From this stage, the out-of-plane displacement field is identical in format to that acquired from laser vibrometry used in experimental study.

Damage was introduced by performing a Boolean subtraction to replicate the saw used in experimental studies. A $5 \times 1 \mathrm{~mm}$ rectangle is subtracted from the FCL as shown in Figure 6. A mesh spacing of $1 \mathrm{~mm}$ is used in all models. Given the A0 mode will have the highest spatial frequency (approximately $640 \mathrm{rad} / \mathrm{m}$ ), this mesh spacing gives a node to wavelength ratio of 9.82 . Propagating stress waves are

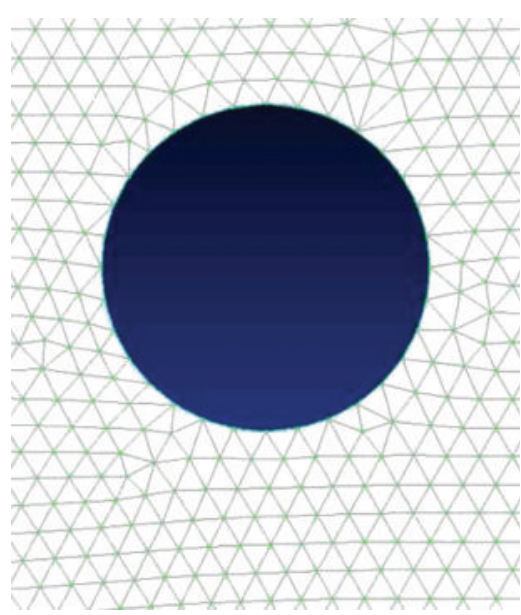

(a) Undamaged

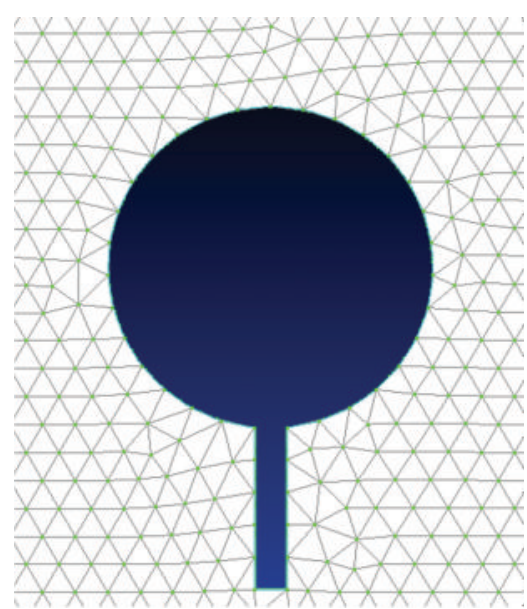

(b) Damaged

Figure 6: Fuel vent holes.

solved using the transient dynamic/time history solver. A time step is set to be 0.2 us. The total time record calculated is 100 microseconds. This time record was selected to be long enough for the wave packet to travel downstream, towards the edges of specimen.

In this computational study, the size of the actuation region on the model is identical to the PZT used in experimental study. The excitation scheme used to produce a Lamb wave caused by the low-profile PZT is similar to that described by Constantin et al. [8]. Figure 7 shows the 2 types of input forces placed on the FE geometry. The plane of the plate is described by the $X-Y$ plane, and the $Z$ axis is out-ofplane and perpendicular to the plate.

4.2. Results. Displacement fields were solved for 4 models. Flawless and damaged models were made in the T beam's original and modified form. Equation (1) is applied to the flawless and damaged displacement fields to create the scattered field. The displacement fields were analysed for a suitable time window which the waves affected by the defect 


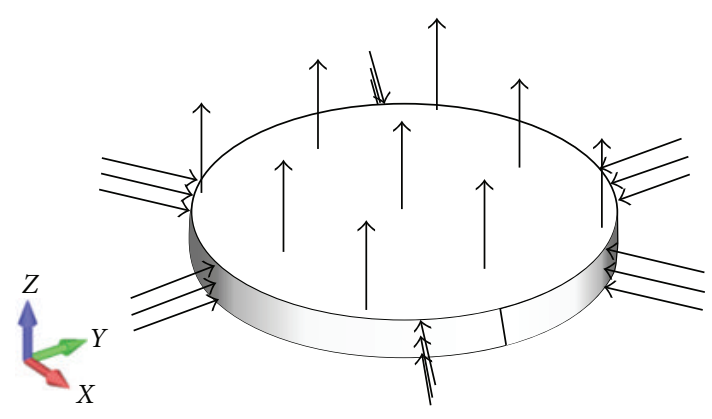

Figure 7: Excitation scheme employed in FEA.

TABle 1: Comparison of sensitivity to damage before and after modification.

\begin{tabular}{ll}
\hline \multicolumn{2}{c}{ Improvement (FEA) } \\
\hline Original: maximum scatter on top surface (nm) & 1.18 \\
Modified: maximum scatter on top surface $(\mathrm{nm})$ & 2.02 \\
Percentage improvement $(\%)$ & 71.2 \\
\hline
\end{tabular}

would propagate to the flange. The window chosen for this is between $50-85$ us.

The side-by-side comparison is shown in Figure 8 and displays obvious benefits from the addition of the reflector. Scatter intensities are visibly larger in the modified specimen for the same damage size, indicating that sensitivity to damage has been improved.

In the original specimen, the area sensitive to damage is located on the opposite side of the T-beam $(x=100 \mathrm{~mm})$. Therefore, in order to achieve acceptable detectability a second sensor must be placed in that vicinity, in a similar fashion to the pitch-catch arrangement used in traditional ultrasonics. However, the modified specimen reveals a very sensitive area where the PZT actuator has already been located. As the time window for excitation (0-20 us) and time window for reception (50-85 us) do not clash it is highly probable that a single transducer can be used for transmission and reception, thereby saving weight and cost.

The improvement can be measured more quantitatively by measuring scatter intensity at feasible points of detection. As explained earlier, placement of sensors is constrained to the top surface due to the presence of fuel in a wet wing. Therefore, only sensitive locations on the top surface will be used to gauge sensitivity to damage in the FCL.

Table 1 summarises the improvement in defect detectability when monitoring the top surface. A significant $(71 \%)$ increase in sensitivity to damage is observed when comparing the modified design to the original. This demonstrates that the directed and precisely focused energy at the FCL is advantageous in detection of damage.

\section{Experimental Study}

5.1. Lamb Wave Transmission and Reception. Transmission was achieved using piezoelectric transducers (PZT), while the Lamb waves were received using a laser vibrometer. A movable table allows positioning of the laser's scan point. This simple concept can be visualised in Figure 9. If the scan is repeated thousands of times across the entire specimen a complete displacement field can be captured. A rig enabling this has been assembled for Lamb wave imaging shown in Figure 9.

Laser vibrometry has been a key tool to aid comprehension and understanding of Lamb waves as shown by Staszewski et al. [9] and Rajic and Rosalie [10]. A laser is used to detect Lamb wave displacements in a specimen rather than a PZT. This noncontact approach is more precise and consistent due to the removal of coupling mediums and the pin point accuracy of the laser. The major advantage of this technique is the speed and potential for automation due to the noncontact operation.

A low-profile PZT is used, $10 \times 1 \mathrm{~mm}$ in diameter and thickness, respectively. It is produced using "Pz26" material sourced from Ferroperm. Coupling to the specimen is done by Araldite epoxy, while the electrical connections to the PZT are taken care of by conductive copper adhesive strips. This is done in order to maintain a low-profile and avoid heat stress involved with soldering. Figure 9 demonstrates an example.

An excitation signal was generated comprising of sine waves enclosed in a Hanning window function. A resonant frequency of $240 \mathrm{kHz}$ was chosen for this. This helped combat the notorious signal to noise ratios associated with laser vibrometers. Furthermore, 32 ensemble averages are taken to further reduce noise. The PZT was excited through a Krohn-Hite model 7602 broadband amplifier set to deliver peak-to-peak amplitude of $50 \mathrm{~V}$. Out of plane displacement is detected by a Polytec OFV 505 laser vibrometer. Input (displacement) and output (excitation) data is sampled at 10 and $4 \mathrm{MHz}$, respectively. These rates are the maximum allowed by the National Instruments PCI-6115 data acquisition card. Motion is taken care of by a computer control $X-Y$ table equipped with stepper motors. The ratio of the table is 5000 steps per $\mathrm{mm}$ providing $0.0002 \mathrm{~mm}$ resolution.

The rig has been programmed to collect data in a zigzag pattern to minimise wasted motion. The data is then rearranged to form a grid such that a complete image of the plate displacement field can be formed. Scan points on our rig are set to $1.5 \mathrm{~mm}$ spacing giving a point density of 1 point per $2.25 \mathrm{~mm}^{2}$. This is a great increase in density compared to the 1 point per $15.71 \mathrm{~mm}^{2}$ (420 points over $110 \times$ $60 \mathrm{~mm}$ ) reported by Staszewski et al. [11]. Consequently, our resolution is much higher.

Figure 10 shows the fabricated specimens. They have been made with the dimensions specified in the ray trace model. The $\mathrm{T}$ beam has been cut from an aluminium extrusion. A computer numerically controlled (CNC) milling machine was used to cut the reflector using the dimensions from the ray tracing study.

5.2. Results. In order to maintain consistency and allow for comparison, the parameters such as time window of 5085 us selected in the FEA study have been retained in the experimental study. Damage detection has been attempted 


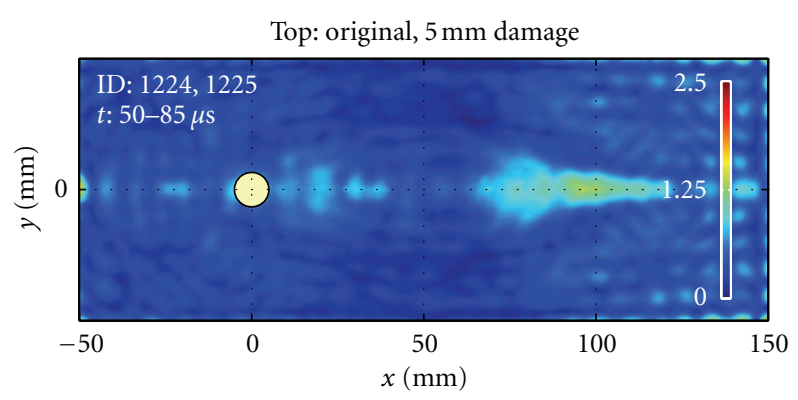

(a)

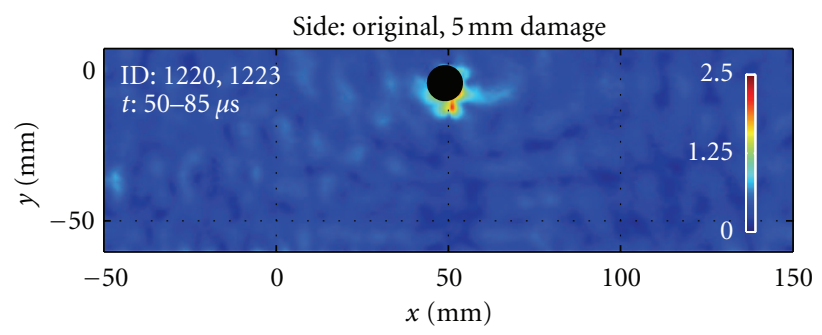

(c)



(b)

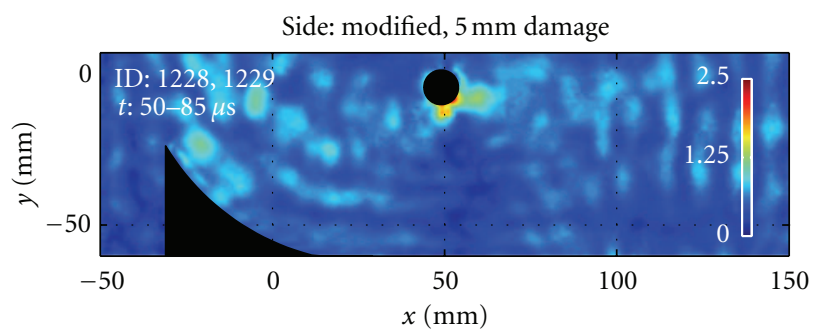

(d)

FIGURE 8: Scatter plots predicted by FEA before and after modification.

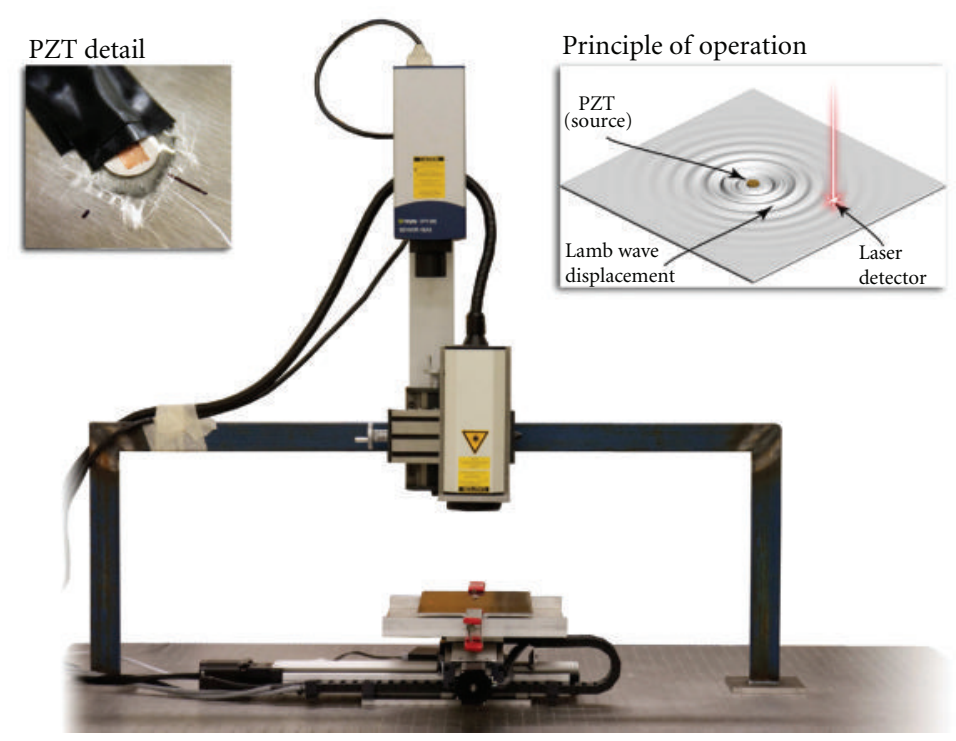

FIGURE 9: Laser vibrometry rig.

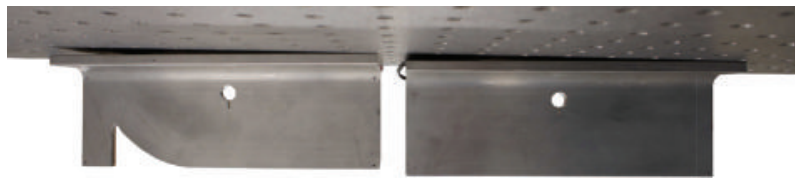

Figure 10: Photo of fabricated specimens.

on $1 \mathrm{~mm}$ damage. Figures $11(\mathrm{a})-11(\mathrm{~d})$ show the resulting scatter field given this damage size. Although only the top surface is available for sensor placement, both surfaces have been scanned and presented as the side surface is important for greater comprehension. Both sides of the original specimen display almost no signs of the $1 \mathrm{~mm}$ damage, making it undetectable.

The modified specimen yields a superior sensitivity to the $1 \mathrm{~mm}$ damage as shown in Figures 11(b) and 11(d). Large amounts of scatter are revealed by the side scan, which are not present in the original specimen. Consequently, a highsensitivity is implied; however, only the results on the top edge of the side scan $(z=0 \mathrm{~mm})$ are relevant to the top 


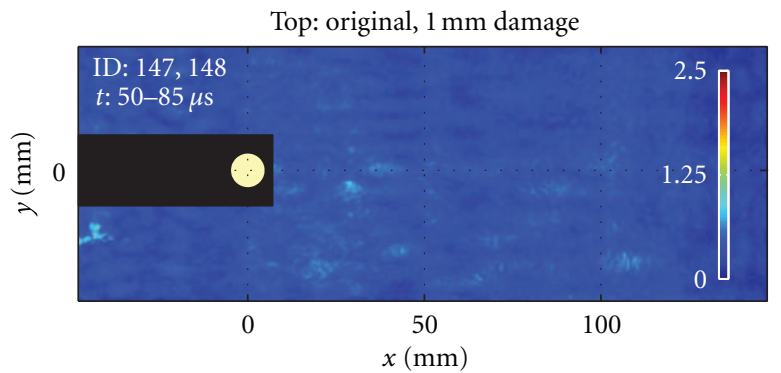

(a)



(c)

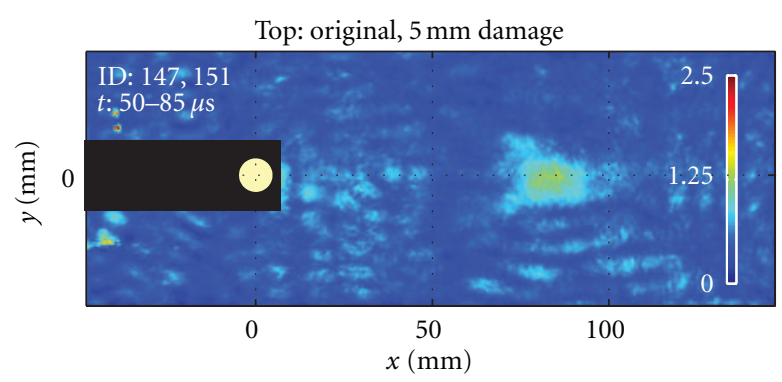

(e)



(g)



(b)

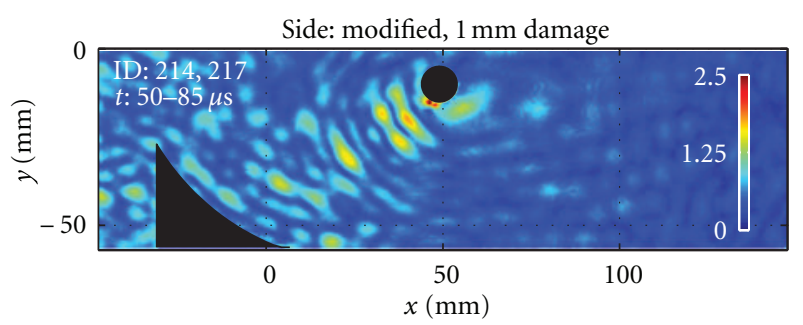

(d)

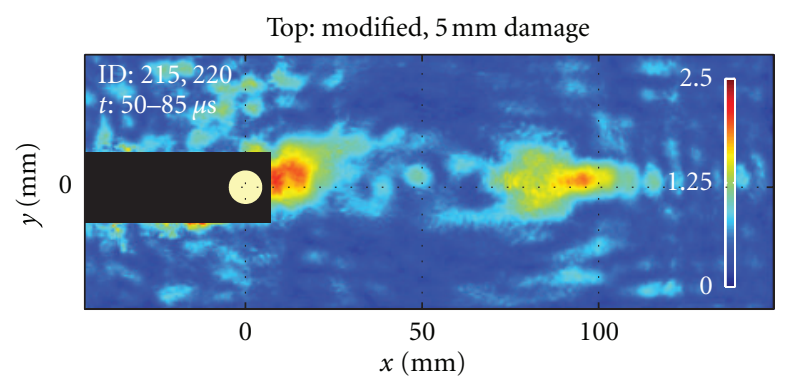

(f)



(h)

FIGURE 11: Scatter plots generated from experimental scans before and after modification.

surface used in detection. Along this edge, the sensitivity is greatest when $x<0 \mathrm{~mm}$; however, this area cannot be analysed due to the supporting lead wires for the PZT blocking the laser. The right half of the specimen $(x>$ $50 \mathrm{~mm}$ ) shows small amounts of scatter, this leaves an area between $x=0 \mathrm{~mm}$, to $x=50 \mathrm{~mm}$ as the best possibility for damage detection. Figure 11(b) confirms a location $(x=$ $10 \mathrm{~mm}, y=0 \mathrm{~mm}$ ) as the location of highest sensitivity.

This indicates that the modified specimen not only redirects the incident waves towards the defects but also focuses the reemitted waves from the defect towards the top surface where the PZT resides. It is highly likely that higher sensitivities would be achieved where the PZT actuator is placed; however, it is not possible to scan this area. Despite this the arrangement is similar to the pulse-echo setup used in classical ultrasonics [12]. Hence, interrogation of the structure can be performed with a single PZT given the right hardware.

Figures $11(\mathrm{e})-11(\mathrm{~h})$ show the results when $5 \mathrm{~mm}$ of damage has been introduced. At $5 \mathrm{~mm}$, the damage can be detected in both original and modified specimens. However, a much higher sensitivity to the damage is observed in the modified specimen. The location of highest sensitivity remains the same for the modified specimen, whereas the original specimen did not have a location. The increase to $5 \mathrm{~mm}$ damage length gives rise to a high-sensitivity location 


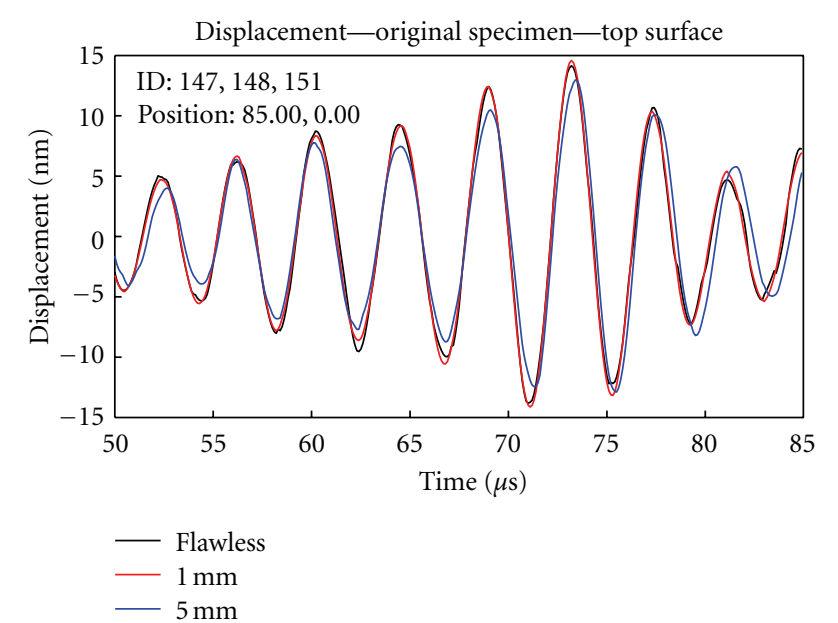

FIGURE 12: Displacement at maximum sensitivity location on original specimen.

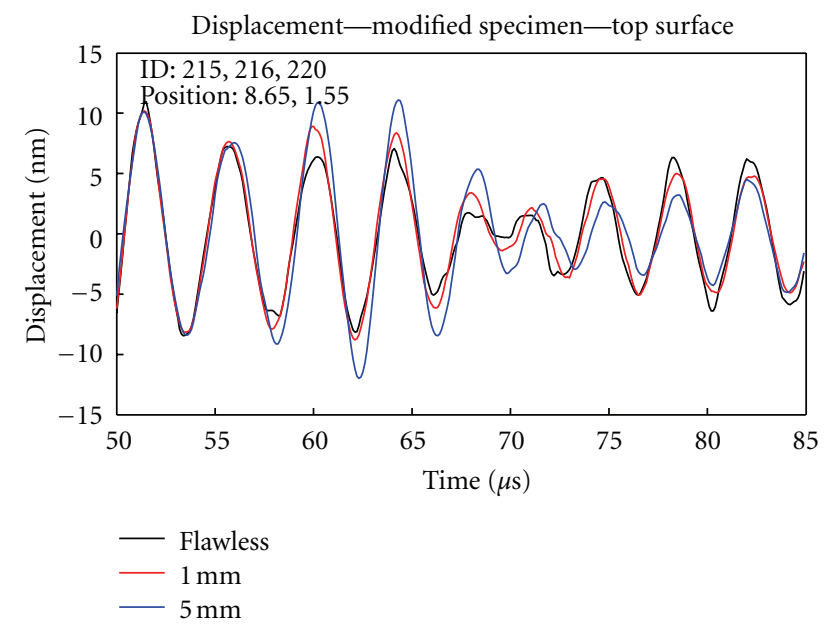

FIGURE 13: Displacement at maximum sensitivity location on modified specimen.

on the right side of the original specimen $(x=85 \mathrm{~mm}$, $y=0 \mathrm{~mm}$ ).

Table 2 summarises and quantifies the results from this section. As in the numerical study, the maximum scatter in the top surfaces were compared to measure the improvement gained by modifying the specimen. The experiments reveal a $76 \%$ and $62 \%$ improvement for the 1 and $5 \mathrm{~mm}$ damages, respectively. These figures are in line with the improvement predicted by the numerical study.

The displacement time series in Figures 12 and 13 demonstrate sensitivity of the Lamb wave response to damage in greater detail. The location of maximum scatter and sensitivity on the top surface are used in plotting the displacement time series. In the case of the original specimen, this position is ( $x=85 \mathrm{~mm}, y=0 \mathrm{~mm})$ while this is located at $(x=8.65 \mathrm{~mm}, y=1.55 \mathrm{~mm})$. The original specimen shows very little deviation from the flawless waveform. This makes it impossible to discriminate the response to $1 \mathrm{~mm}$ damage from that of a flawless specimen. Conversely, the
TABLE 2: Comparison of sensitivity to damage before and after modification.

\begin{tabular}{lcc}
\hline \multicolumn{3}{c}{ Improvement (experimental) } \\
\hline $\begin{array}{l}\text { Cut size (mm) } \\
\begin{array}{l}\text { Original: maximum scatter on } \\
\text { top surface (nm) }\end{array}\end{array}$ & 1 & 5 \\
$\begin{array}{l}\text { Modified: maximum scatter on } \\
\text { top surface (nm) }\end{array}$ & 0.58 & 1.283 \\
Percentage improvement (\%) & 76.0 & 2.076 \\
\hline
\end{tabular}

modified specimen shows a significantly different response from flawless even at $1 \mathrm{~mm}$ damage size indicating a greater sensitivity to damage.

\section{Conclusions}

This work has proposed a different approach to SHM, in which the structure becomes another parameter to optimize. This new parameter has been optimized through ray tracing to arrive at a design which redirects Lamb wave energy towards the desired inspection area which is placed in a hard to-reach-location. Using FEA to confirm the efficacy of the method, it was determined that around 70\% improvement in sensitivity could be achieved from optimization. Following the numerical study, an experimental study confirmed the improvement. Additionally, the $1 \mathrm{~mm}$ defect could only be detected by the modified specimen. This improvement in detectability is highly desirable in low tolerance structures.

It has been noted that the modification presented here would not be practical due to the amount of material removal inhibiting the structure from carrying its original load. However, the specimen is only used as a demonstration of the optimization concept. The concept is expected to be implemented on existing boundaries rather than making new ones. An example of such a specimen is shown in work by Weller and McDonald [13]. The F-111 wing pivot has stiffeners which have an existing taper which may be optimized for Lamb wave redirection.

\section{Acknowledgment}

The authors wish to acknowledge the financial support provided by USAF AOARD-09-4036.

\section{References}

[1] B. C. Lee and W. J. Staszewski, "Modelling of Lamb waves for damage detection in metallic structures: Part I. Wave propagation," Smart Materials and Structures, vol. 12, no. 5, pp. 804-814, 2003.

[2] N. Rajic, S. C. Galea, and W. K. Chiu, "Autonomous detection of crack initiation using surface-mounted piezotranducers," Smart Materials and Structures, vol. 11, no. 1, pp. 107-114, 2002.

[3] Y. L. Koh and W. K. Chiu, "Numerical study of detection of disbond growth under a composite repair patch," Smart Materials and Structures, vol. 12, no. 4, pp. 633-641, 2003. 
[4] I. A. Viktorov, Rayleigh and Lamb Waves: Physical Theory and Applications. Ultrasonic Technology, Plenum Press, New York, NY, USA, 1967.

[5] M. Castaings and B. Hosten, "Guided waves propagating in sandwich structures made of anisotropic, viscoelastic, composite materials," Journal of the Acoustical Society of America, vol. 113, no. 5, pp. 2622-2634, 2003.

[6] W. H. Ong and W. K. Chiu, "Damage quantification in plates using lamb waves," in Proceedings of the Australasian Congress on Applied Mechanics, Perth, Australia, 2010.

[7] W. H. Ong and W. K. Chiu, in Proceedings of the Asia Pacific Workshop on Structural Health Monitoring, Tokyo, Japan, 2010.

[8] N. Constantin, S. Sorohan, M. Gǎvan, and V. Raetțchi, "Efficient and low cost PZT network for detection and localization of damage in low curvature panels," Journal of Theoretical and Applied Mechanics, vol. 49, no. 3, pp. 685-704, 2011.

[9] W. J. Staszewski, B. C. Lee, L. Mallet, and F. Scarpa, "Structural health monitoring using scanning laser vibrometry: I. Lamb wave sensing," Smart Materials and Structures, vol. 13, no. 2, pp. 251-260, 2004.

[10] N. Rajic and S. C. Rosalie, A Feasibility Study into the Active Smart Patch Concept for Composite Bonded Repairs, Defence Science and Technology Organisation, Melbourne, Australia, 2008.

[11] W. J. Staszewski, B. C. Lee, and R. Traynor, "Fatigue crack detection in metallic structures with Lamb waves and 3D laser vibrometry," Measurement Science and Technology, vol. 18, no. 3, pp. 727-739, 2007.

[12] NASA Ultrasonic Testing of Aerospace Materials.

[13] S. Weller and M. McDonald, Stress Analysis of the F-111 Wing Pivot Fitting, Defence Science and Technology Organisation, Melbourne, Australia, 2000. 

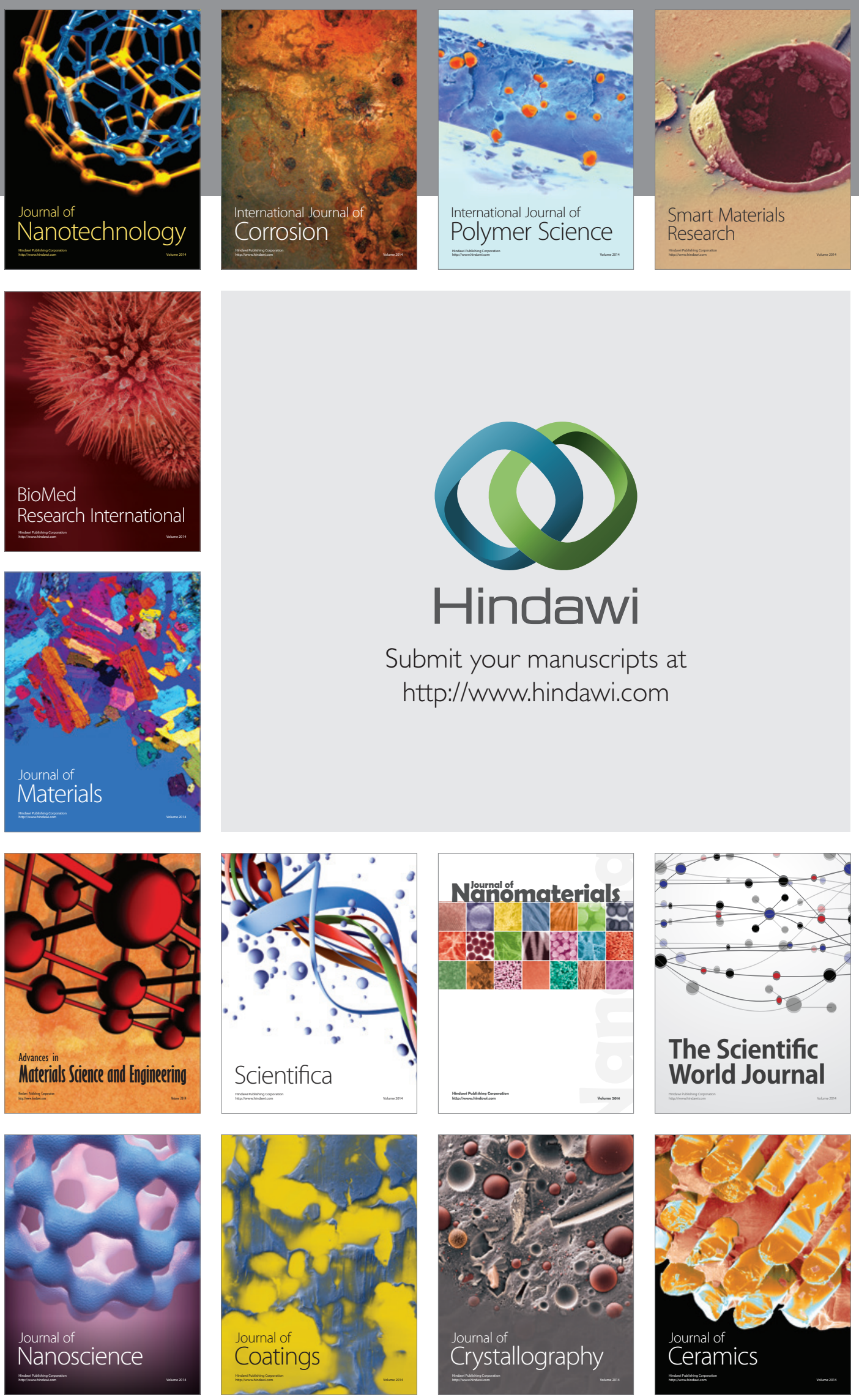

The Scientific World Journal

Submit your manuscripts at

http://www.hindawi.com

\section{World Journal}

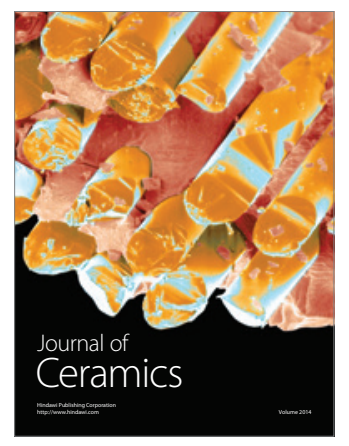

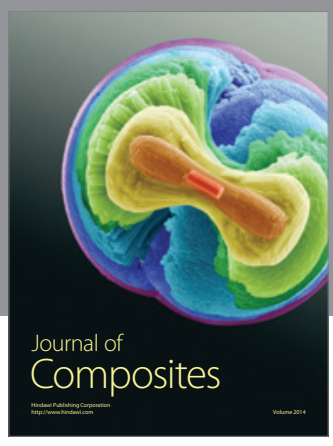
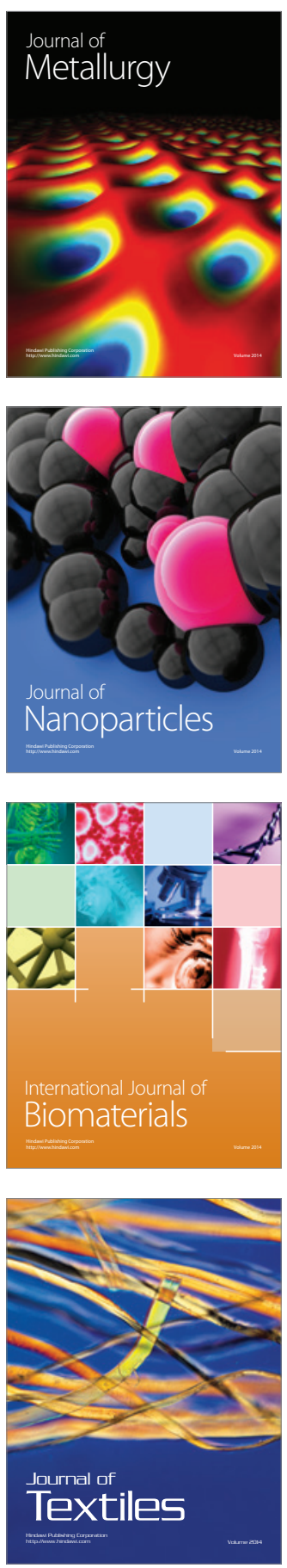